\title{
MAGNETIC FIELDS IN MOLECULAR CLOUD CORES
}

\author{
Z.P. ZHOU ${ }^{1}$ and X.W. ZHENG ${ }^{2}$ \\ ${ }^{1}$ Division of Radioastronomy, Purple Mountain Observatory, \\ Nanjing, China \\ ${ }^{2}$ Department of Astronomy, Nanjing University, Nanjing, China
}

Strong circular polarization of $\mathrm{OH}$ masers at 1665 and $1667 \mathrm{MHz}$ lines has been observed towards the molecular cloud cores associated with HII regions. Magnetic field strengths of a few $\mathrm{mG}$ are derived from the Zeeman splitting of $\mathrm{OH}$ lines. For instance, a magnetic field of about $4 \mathrm{mG}$ in the masing region of $\mathrm{W} 3(\mathrm{OH})$ has been estimated by $\mathrm{OH}$-line Zeeman splitting (Davies, 1974). VLBI observations show that the OH maser spots project onto or very close to the surface of associated compact HII regions (Reid et al., 1986). The observational evidence demonstrates that the scales of $\mathrm{OH}$ maser components surrounding a compact HII region ( $\mathrm{R}$ $10^{16} \mathrm{~cm}$ ) are about $10^{14} \mathrm{~cm}$ in diameter with an amplification pathlength of $\sim 10^{15} \mathrm{~cm}$. Hence the magnetic fields determined by the Zeeman splitting of $\mathrm{OH}$ maser lines appear partly very close to the associated HII region. Elitzur (1979) has theoretically obtained similar results as above.

The Stokes parameter V spectra of non-masing $\mathrm{OH}$ lines in emission and in absorption towards some molecular clouds have been observed by Kazès and Crutcher (1986). Magnetic field strengths given in Table 1

Table 1. Magnetic field strengths derived from the measurements of nonmasing $\mathrm{OH}$ Stokes parameter $\mathrm{V}$ in emission and in absorption towards molecular cloud cores.

\begin{tabular}{|c|c|c|c|c|c|c|c|c|}
\hline \multirow[t]{2}{*}{ Source } & \multirow{2}{*}{$\begin{array}{l}\text { Emission/ } \\
\text { absorption }\end{array}$} & \multicolumn{5}{|c|}{ B $(\mu G)^{*}$} & \multirow[t]{2}{*}{$\log B$} & \multirow{2}{*}{$\log n$} \\
\hline & & 166 & $5 \mathrm{MHz}$ & 166 & $7 \mathrm{MHz}$ & average & & \\
\hline NGC 2024 & A & +39 . & $3 \pm 1.1$ & +36 & \pm 1.8 & $+37.7 \pm 1.5$ & 1.58 & 4.9 \\
\hline W22 & A & -14 & $5 \pm 1.8$ & -20 & $5 \pm 1.4$ & $-17.5 \pm 1.6$ & 1.24 & 3.0 \\
\hline W3 & A & +65 & \pm 20 & +74 & \pm 8 & $+69.5 \pm 14$ & 1.84 & 5.0 \\
\hline L134 & $\mathbf{E}$ & -8 & \pm 8 & -22 & \pm 8 & $-15 \quad \pm 8$ & 1.18 & 4.0 \\
\hline$\rho$ Oph & $\mathbf{E}$ & -17 & \pm 7 & -48 & \pm 24 & $-32.5 \pm 21$ & 1.51 & 4.5 \\
\hline W40 & A & -8 & \pm 4 & -5 & \pm 5 & $-6.5 \pm 4.5$ & 0.81 & 4.5 \\
\hline W51 & A & -4 & \pm 7 & -5 & \pm 13 & $-4.5 \pm 10$ & 0.65 & 3.0 \\
\hline L889 & A & -7 & \pm 8 & -9 & \pm 9 & $-8 \pm 8.5$ & 0.90 & 3.5 \\
\hline TMC1 & E & +18 & \pm 7 & +35 & \pm 14 & $+26.5 \pm 10.5$ & 1.42 & 4.5 \\
\hline
\end{tabular}

${ }^{*}$ A positive magnetic field is directed away from the observer.

291

R. Beck et al. (eds.), Galactic and Intergalactic Magnetic Fields, 291-292.

(C) 1990 IAU. Printed in the Netherlands. 
emerge from more extended parts of molecular clouds than the masing regions. Average magnetic field strengths derived from 1665 and 1667 MHz are adopted in our Table.

A plot of $\log B$ against $\log n$ is shown in Figure 1 where $B$ is the magnetic field strength of the molecular cloud in $\mu \mathrm{G}$ along the line of sight and $n$ is the gas density in $\mathrm{cm}^{-3}$. The dashed line in Figure 1 denotes the fitting line of $B \sim n^{1 / 2}$ theoretically predicted by Mouschovias (1983) with a background field value of $5 \mu G$ (Kazès and Crutcher, 1986). What is plotted as the theoretical line is one-half of the full field value in order to account statistically for the fact that the Zeeman measurement is sensitive only to the line-of-sight component of the magnetic field; on average, the true field will be twice the value measured in observations. Following Mouschovias (1983) during contraction the field strength $B$ in a cloud core increases with gas density $n$ as $B / B_{0}=\left(n / n_{0}\right)^{1 / 2}$ where the quantities $B_{0}$ and $n_{0}$ refer to values at which gravitational forces become important. In this case we adopt $B_{0}=5 \mu G$ and $n_{0}=250 \mathrm{~cm}^{-3}$. If $B_{0}=3 \mu \mathrm{G}$, then $n_{0}=87 \mathrm{~cm}^{-3}$. The corresponding masses of clouds are $22 \mathrm{M}_{\odot}$ and $39 \mathrm{M}_{\odot}$, respectively. These masses are small, but are not too small to be acceptable (Benson, 1986).

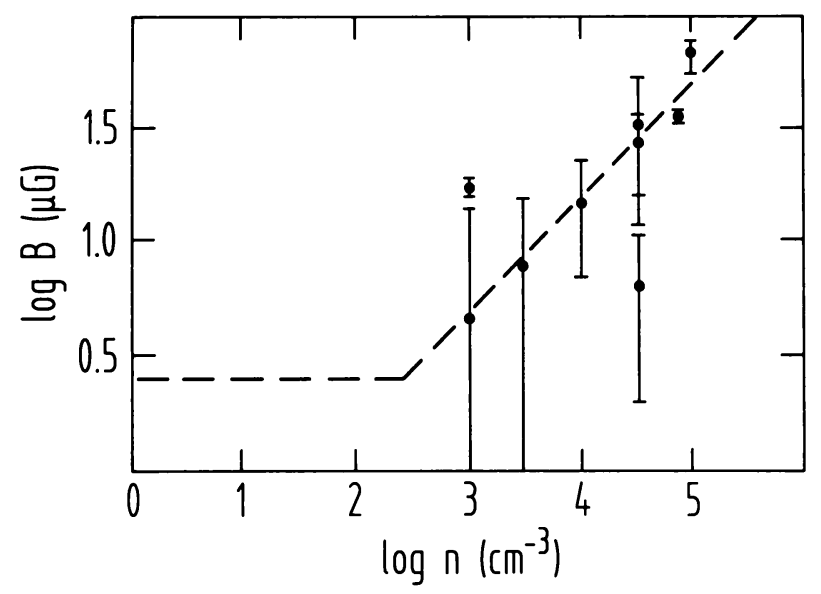

Figure 1. Magnetic field strengths along the line of sight in molecular clouds plotted against gas densities. The dashed line is a fitting line of $B \sim n^{1 / 2}$ theoretically predicted by Mouschovias for a background magnetic field value of $5 \mu \mathrm{G}$ and $a$ cloud mass of $22 \mathrm{M}_{\odot}$.

\section{References}

Benson, P.J. (1986) in A.D. Haschick (ed.), Masers, Molecules and Mass Outflows in Star Forming Regions, Haystack Observatory, p. 55.

Davies, R.D. (1974) in F.J. Kerr and S.C. Simonson III. (eds.), Galactic Radio Astronomy (IAU Symp. No. 60), Reidel, Dordrecht, p. 275.

Elitzur, M. (1979) Astron. Astrophys. 73, 322.

Kazès, I. and Crutcher, R.M. (1986) Astron. Astrophys. 164, 328.

Mouschovias, T.Ch. (1983) in J. Stenflo (ed.), Solar and Stellar Magnetic Fields (IAU Symp. No. 102), Reidel, Dordrecht, p. 479.

Reid, M.J., Myers, P.C. and Bieging, J.H. (1986) Center for Astrophysics Preprint No. 2341. 